\title{
A Geometrical Construction with Physical Applications.
}

\author{
By W. Peddie.
}

The Diagram shows two complete, and one partially complete, concentric circles. The radii of the largest and smallest are as four to one respectively.

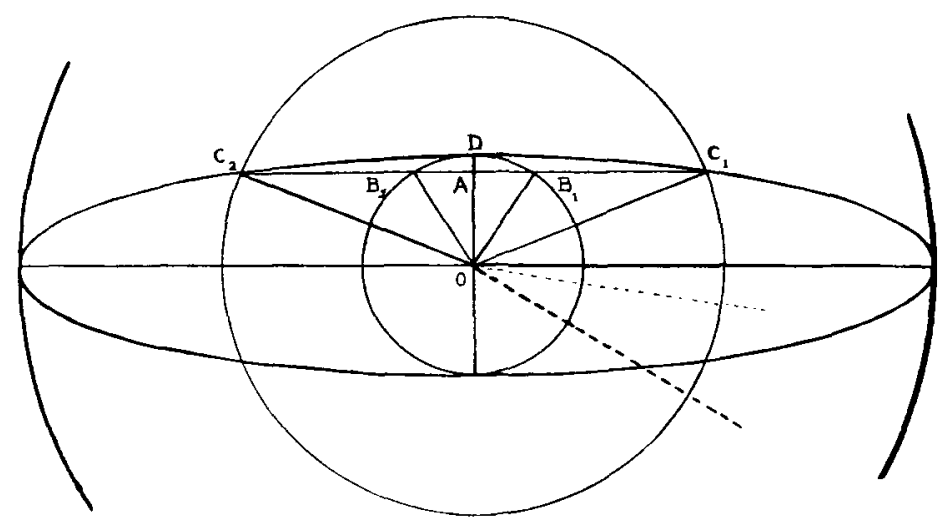

Consider the two circles $B$ and $C$. Let the radius, $O D$, of the smallest be unity, while that of the second is $\mu$. If $A B_{1} O$ be called $i$, while $A C_{1} O$ is $\gamma$, we at once have the relation $\sin i=\mu \sin \gamma$. Thus, if $i$ be an angle of incidence of light on a refracting surface of a medium of respective index $\mu$, then $\gamma$ is the corresponding angle of refraction; and the angle $B_{1} O C_{1}$ is $i-\gamma$.

If now we vary $i$ and $\gamma$ while keeping $C_{2} A C_{1}$ parallel to itself it is easy to see that $i-\gamma$ increases at a greater and greater rate as $i$, say, is steadily increased. For, when $i$ is very small, $B C$ is very nearly constant. And $B C$ increases at a greater and greater rate as $i$ increases steadily. So, in the triangle $B_{1} O C_{1}$, the lengths of the two sides are constant while the base increases at a greater and greater rate as the angle at $O$ opens out through $A B_{1} C_{1}$ moving upwards while preserving its direction. Therefore $i-\gamma$ increases at a greater and greater rate as $i$ increases steadily.

The angle $i--\gamma$ is the angle of deviation produced by the first refraction-say $i_{1}-\gamma_{1}$. If now the light passes out at a second surface (as of a prism) the angle $i_{2}-\gamma_{2}$ is added to the first deviation. 
Let us make $i_{1}=i_{2}$, and therefore $\gamma_{1}=\gamma_{2}$. If we now, keeping the path of the incident ray fixed, increase $i$ by turning the prism, we necessarily decrease $i_{2}$. So we have increased $i_{1}-\gamma_{1}$ and diminished $i_{2}-\gamma_{2}$. But, by the above proposition, we have increased $i_{1}-\gamma_{1}$ more than we have decreased $i_{2}-\gamma_{2}$. Therefore the total deviation is increased. This is obviously true if we increased $i_{2}$ and diminished $i_{1}$ from equality. Thus the symmetrical condition gives a state of minimum deviation. In this way the stationary conditions of deviation which occur in the production of halos and rainbows can be simply explained.

The same construction, with the associated ellipse (principal radii in ratio $4: 1$ ), can be used for a magnetic application as follows.

Let the faint dotted line represent the direction of a stable axis of magnetisation in a ferromagnetic crystalline plate cut parallel to a face plane of a cubic crystal. Let also the major diameter of the ellipse and the line $C_{2} B_{2} A B_{1} C_{1}$ be in the direction of magnetisation of the plate. If the angle between the faint dotted line and the major axis be $\theta_{1}$, the construction gives $A B_{1} O=4 \theta_{1}$ and $A C_{1} O={ }_{1} \theta_{0}-\theta^{1}$, where ${ }_{1} \theta_{0}$ is the angle between $O C_{1}$ and the faint dotted line. In that case $O C_{1}=h$ gives the direction and magnitude of the external magnetic field which gives rise to the magnetisation, and is just sufficient to bring the molecular magnets to the state of instability from which, with dissipation of magnetic energy, they flash round to take up a new position of stable equilibrium under the same field $O C_{1}$. The unit in which $h$ is measured is here the maximum value of the component of the internal magnetic field transverse to the direction of magnetisation, i.e. parallel to $A O$.

If we use a smaller circle than that of radius $O C_{1}$, cutting $B_{2} B_{1}$ at a point $C^{\prime}{ }_{1}$ inside $C_{1}$, then $O C^{\prime}{ }_{1}$ will represent the direction and magnitude of the external field which would maintain stable equilibrium. The construction still holds if $C_{1} C_{2}$ be displaced parallel to itself if the direction of the stable crystalline axis (faint dotted line) be altered correspondingly. In fact, quite generally, if the point $C^{\prime}{ }_{1}$ lies within the ellipse, the magnetic configuration so specified is one of stability. If it lies on the ellipse the configuration is just at the limit for stability; and if it lies outside the ellipse, the magnetic configuration is unstable. 